\title{
Schizophrenia in the light of precision medicine: A time for reconsideration
}

\author{
Suresh Sundram
}

Schizophrenia is a severe neurodevelopmental disorder affecting $0.7 \%$ of the global population regardless of nationality, social class, sex or ethnicity (1). It manifests between the ages of 15-25 years, is lifelong, persistent and crippling for most. There are three major symptom domains: positive, negative and cognitive symptoms (2). Psychotic symptoms such as delusions and hallucinations are terrifying and attract medical attention, but at least in part are responsive to treatment with antipsychotic medications. Conversely, negative symptoms such as social withdrawal and poverty of thought and cognitive symptoms such as impaired attention and executive dysfunction are functionally more impairing but have no effective treatments $(3,4)$. Many people with schizophrenia experience a gradual decline of $\geq 10$ IQ points, impaired attention, memory deterioration and a decreased ability to think flexibly or creatively (executive dysfunction) (5). Negative symptoms such as social withdrawal, amotivation and anhedonia leave sufferers lonely, unable to work and often depressed (6). This symptom burden has marked psychosocial impacts, with loss of educational and vocational opportunities and ability, social isolation, reduced fertility and high rates of suicidality, up to 12 times that of the general population $(3,7)$. Additionally, mortality is increased and life expectancy is reduced by approximately 12 years (1). Economic costs include direct medical costs and indirect costs associated with lost productivity; this varies widely globally - from annual societal costs per patient of US dollars 5,818 in Thailand to US dollars 94,587 in Norway (8). The reason for these grim outcomes is because the illness starts in young people, and treatments are only symptomatic and partially effective. What is lacking is a precision medicine approach to schizophrenia, as has occurred in oncology, haematology and other areas of medicine (9). Ultimately, this gap is due to the failure to understand the pathology of schizophrenia, which has prevented the development of diagnostics, and disease modifying, breakthrough treatments, as well as truly preventative measures.

The search for this "holy grail" in the pathology of schizophrenia has focused in the past two decades on large scale genome wide association studies (GWAS). These studies involving many hundreds of thousands of participants have resulted in three main findings. Firstly, much of the genetic architecture of schizophrenia at a population level is due to the combination of possibly thousands of genetic loci, each of small effect generally, within the non-coding region (10). Secondly, there are some highly penetrant de novo protein altering mutations and genic copy number variants which are highly pathogenic but rare; and thirdly, considerable pleiotropy and cross-heritability exists between schizophrenia and other psychiatric disorders such as bipolar disorder, major depression and attention deficit hyperactivity disorder $(11,12)$.

Although it is conceivable that there may be fully penetrant rare genetic mutations, all currently known risk genes must interact with environmental factors to induce schizophrenia. These factors are numerous and include maternal second trimester insults such as infection and starvation, stress, migration and cannabis use (13). The variety of genetic and environmental risk factors in conjunction with the variability in symptom profiles and clinical course strongly argue that there is no single cause of schizophrenia but rather it is a syndrome with multiple possible aetiologies and outcomes (14). The task then becomes how to probe this diversity to disentangle discrete sub-types within the disorder. Numerous approaches have been used based on, for example, symptomatology, cognitive profiles, neuroimaging and blood based biomarkers (15). One easily discernible differentiating characteristic may be to use treatment response to antipsychotic drugs (APD). Moreover, because we have some understanding as to how APD work, this may provide clues as to underlying pathological processes.

Antipsychotic drugs are the linchpins of schizophrenia treatment (2), however, they are not specific for schizophrenia but can be used for any cause of psychosis, for example, bipolar disorder or drug-induced psychosis. Overwhelming data from positron emission tomography, clinical, animal and molecular studies support APD action working through dopamine-D2 receptor antagonism, and there are currently no approved treatments for schizophrenia that do not possess this action (3). Although this is consistent with a central role for dopamine in 
schizophrenia, there are some inconsistencies. Specifically, some $30-40 \%$ of people with schizophrenia do not respond to dopamine-D2 receptor antagonism and this group has been defined as treatment resistant schizophrenia (TRS) (2). There is considerable variation in defining TRS, but current definitions include a failure to respond two trials of chemically different classes of antipsychotic drugs at maximally tolerated doses within the recommended therapeutic range for at least six weeks duration (16). Response is accepted as improvement in positive symptoms, but may include functional improvement. Therefore, treatment responsive patients show improvements in psychotic symptoms to dopamine-D2 receptor antagonism similar to other psychotic disorders, suggesting these disorders collectively demonstrate some level of dopamine dysfunction; whereas, TRS patients may be exhibiting another type of pathology. Identifying putative alternate pathologies for this TRS group may both provide insights into the different subtypes of schizophrenia and open paths for alternative treatments for those who do not respond to conventional dopamine-D2 receptor antagonism.

For TRS, the principal treatment option is clozapine. A recent meta-analysis showed clozapine to be superior to other treatments for TRS but the number needed to treat was 9 (17). Similarly, clozapine is only effective in approximately $50 \%$ of people with TRS, raising the strong possibility that additional treatment sub-groups exist. One such additional sub-group could involve a central role for serotonin. Pimavanserin is a serotonin 5-HT2A/ $2 \mathrm{C}$ receptor agent that was approved in 2016 by the United States Food and Drug Administration (FDA) for the treatment of levo-dopa induced psychosis in Parkinson's disease (18). It has no other appreciable receptor affinity and functions as an inverse agonist at the serotonin 5-HT2A receptor. Initial trials in schizophrenia did not support stand-alone efficacy, but these trials were conducted with non-TRS patients. Much more intriguing though, is a recent finding in non-clozapine responsive TRS patients (19). Here, pimavanserin showed considerable effect in reducing positive symptoms in an open label case series of ten clozapine non-responsive patients. This finding requires rigorous testing in a blinded controlled trial with clozapine to establish its validity, but nevertheless, supports the proposition that treatment response characteristics could be a useful strategy to disambiguate sub-types in schizophrenia.

However, even within this approach there may exist additional complexity. A recent naturalistic observational cohort study covering the years 1996-2014 compared APD monotherapy and polypharmacy on outcomes in schizophrenia measured as re-hospitalisation (20). This study reported clozapine was involved in 7 of the 8 most effective treatments in reducing re-hospitalisation, with olanzapine combined with long-acting injectable APD (LAI) as the only non-clozapine treatment. Clozapine in conjunction with the partial dopamine-D2 receptor agonist, aripiprazole, was the most effective alongside clozapine and olanzapine and clozapine monotherapy. However, clozapine in conjunction with any LAI or any other oral ranked in the top 8 . This indicates that a partial response to clozapine may be improved by adding a dopamine-D2 receptor antagonist, suggesting that the distinction between clozapine responsive TRS and nonTRS may not be clear-cut.

Another complexity relates to the inadequacy of current nosological approaches, which in the absence of objective markers, is reliant on phenomenology. As noted above, recent GWAS demonstrate considerable significant genetic cross-heritability between schizophrenia and bipolar disorder, major depressive disorder, obsessivecompulsive disorder, autism spectrum disorder, anorexia nervosa and attention deficit hyperactivity disorder. These cross-heritabilities, which were up to 0.70 , align with an alternative diagnostic approach such as described by the B-SNIP (Bipolar-Schizophrenia Network for Intermediate Phenotypes) and other groups $(12,15)$. This proposes that patients should be clustered according to objective markers such as genetic, neurophysiological, cognitive, blood based and neuroimaging measures, independent of symptoms. These approaches argue that biological commonalities exist between patients that transcend symptomatic differences. If this was applied to the non-TRS / clozapine responsive TRS / clozapine non-responsive TRS classification proposed above, then it may well be that a proportion of patients with other psychotic disorders such as bipolar disorder and major depression may fit into these groups based on their biological signature. This raises the possibility that a proportion may then respond to clozapine because they share this biological commonality with the clozapine responsive TRS group.

The application of precision medicine approaches to psychotic disorders is well overdue, with no current diagnostic tests, disease modifying treatments or preventative measures. Schizophrenia, as the archetypal psychotic disorder, exhibits extraordinary genetic and environmental heterogeneity indicative of widely variable aetiologies and pathologies. Disentangling this has been deeply problematic, but a simple clinically relevant approach based on treatment response may be a first step. The ability of clozapine to treat a proportion of patients not responsive to conventional APD demarcates a cohort, which when investigated may display an alternate pathology to dopamine-D2 receptor antagonist responsive illness. Similarly, if substantiated, a cohort of clozapine non-responsive patients who improve with serotonin 5-HT2A receptor antagonism (or inverse 
agonism) may exist. Despite these simple categorisations, it is very plausible that the pathology underlying these groups may also be present in other psychotic disorders, opening the possibility of shared treatment strategies. It is evident that the current paradigms and taxonomy for psychotic disorders need to be reconsidered in the light of more sophisticated biological data.

\section{Conflicts of interest}

None declared

Suresh Sundram, Monash University, Department of Psychiatry, School of Clinical Sciences and Monash Health, Australia.

Corresponding Author: S Sundram

Email: Suresh.Sundram@monash.edu

https://orcid.org/0000-0002-9674-0227

\section{References}

1. McGrath J, Saha S, Chant D, Welham J. Schizophrenia: a concise overview of incidence, prevalence, and mortality. Epidemiol Rev 2008; 30: 67-76.

2. Sundram S, Joyce PR, Kennedy MA. Schizophrenia and bipolar affective disorder: perspectives for the development of therapeutics. Curr Mol Med 2003; 3(5): 393-407.

3. Owen MJ, Sawa A, Mortensen PB. Schizophrenia. Lancet 2016; 388(10039): 86-97.

4. Green MF. Impact of cognitive and social cognitive impairment on functional outcomes in patients with schizophrenia. J Clin Psychiatry 2016; 77(2): 8-11.

5. Wells R, Swaminathan V, Sundram S, et al. The impact of premorbid and current intellect in schizophrenia: cognitive, symptom, and functional outcomes. NPJ Schizophr 2015; 1: 15043 .

6. Helfer B, Samara MT, Huhn M, et al. Efficacy and safety of antidepressants added to antipsychotics for schizophrenia: A systematic review and meta-Analysis. Am J Psychiatry 2016; 173(9): 876-86.

7. Morgan VA, Waterreus A, Jablensky A, et al. People living with psychotic illness in 2010: the second Australian national survey of psychosis. Aust N Z J Psychiatry 2012; 46(8): 735-52.

8. Jin H, Mosweu I. The Societal Cost of Schizophrenia: A Systematic Review. Pharmacoeconomics 2017; 35(1): $25-42$.

9. Insel TR. The NIMH Research Domain Criteria (RDoC) Project: precision medicine for psychiatry. Am J Psychiatry 2014; 171(4): 395-7.

10. Sullivan PF, Daly MJ, O'Donovan M. Genetic architectures of psychiatric disorders: the emerging picture and its implications. Nat Rev Genet 2012; 13(8): 537-51.

11. Birnbaum R, Weinberger DR. Genetic insights into the neurodevelopmental origins of schizophrenia. Nat Rev Neurosci 2017; 18(12): 727-40.

12. Brainstorm Consortium, Anttila V, et al. Analysis of shared heritability in common disorders of the brain. Science 2018; 360(6395): pii: eaap8757.

13. Brown AS. The environment and susceptibility to schizophrenia. Prog Neurobiol 2011; 93(1): 23-58.

14. Green, IW, Glausier JR. Different Paths to Core Pathology: The Equifinal Model of the Schizophrenia Syndrome. Schizophr Bull 2016; 42(3): 542-9.

15. Clementz BA. Identification of Distinct Psychosis Biotypes Using Brain-Based Biomarkers. Am J Psychiatry 2016; 173(4): 373-84.

16. Elkis H, Buckley PF. Treatment-Resistant Schizophrenia. Psychiatr Clin North Am 2016; 39(2): 239-65.

17. Siskind D, McCartney L, Goldschlager R, Kisely S. Clozapine v. first- and second-generation antipsychotics in treatment-refractory schizophrenia: systematic review and meta-analysis. Br J Psychiatry 2016; 209(5): 385-92.

18. Sahli ZT, Tarazi FI. Pimavanserin: novel pharmacotherapy for Parkinson's disease psychosis. Expert Opin Drug Discov 2018; 13(1): 103-10.

19. Nasrallah HA, Fedora R, Morton R. Successful treatment of clozapine-nonresponsive refractory hallucinations and delusions with pimavanserin, a serotonin 5HT-2A receptor inverse agonist. Schizophr Res 2019; pii: S09209964(19)30078-7.

20. Tiihonen J, Taipale H, Mehtälä J, Vattulainen P, Correll CU, Tanskanen A. Association of Antipsychotic Polypharmacy vs Monotherapy With Psychiatric Rehospitalization Among Adults With Schizophrenia. JAMA Psychiatry 2019; 20. 